\title{
Partially Transparent Jaumann-Like Absorber Applied to a Curved Structure
}

\author{
Alireza Motevasselian and B. L. G. Jonsson \\ Electromagnetic Engineering Laboratory, School of Electrical Engineering, Royal Institute of Technology (KTH), \\ 10044 Stockholm, Sweden \\ Correspondence should be addressed to Alireza Motevasselian, alireza.motevasselian@ee.kth.se
}

Received 12 April 2011; Accepted 30 June 2011

Academic Editor: Dau-Chyrh Chang

Copyright ( 2011 A. Motevasselian and B. L. G. Jonsson. This is an open access article distributed under the Creative Commons Attribution License, which permits unrestricted use, distribution, and reproduction in any medium, provided the original work is properly cited.

\begin{abstract}
A Jaumann absorber with its metal backing replaced with a combined low-pass and polarizer FSS is investigated with respect to its absorption and its polarization-dependent low-frequency transparency properties. This structure is applied to an idealized curved wing-front end, and its monostatic radar cross-section is determined. The FSS-Jaumann structure preserves an absorption similar to the planar Jaumann absorber in the higher frequency interval and enables a partial transparency in the $\mathrm{TE}_{z}$ polarization at $1 \mathrm{GHz}$. In addition, once the structure is applied to the wing-front end, a significant reduction in two-dimensional radar crosssection for both the $\mathrm{TM}_{z}$ and $\mathrm{TE}_{z}$ polarization over $2-16 \mathrm{GHz}$ is observed. A sensitivity analysis shows that the resistivity of the inner resistive layer has a large impact on the $1 \mathrm{GHz}$ transmission.
\end{abstract}

\section{Introduction}

This paper is a second step towards a design of a curved absorber at high frequencies with a polarization-sensitive transparent window at low frequencies. The structure is intended to be applied to the front end of an aircraft wing. The first step was achieved in [1] where it was shown that a Jaumann-similar absorber could be used to efficiently reduce the monostatic radar cross-section (RCS). This reduction persists over $a \pm 20^{\circ}$ angular interval around the forward direction of an idealized airplane wing. Our long-term goal is to design a curved antenna radome with absorbing properties with low monostatic RCS at high frequencies and a transparent polarization-sensitive antenna window at low frequencies. The purpose of this paper is to investigate the absorption and transparency properties of a Jaumann absorber when the back-metal layer is replaced with a lowpass frequency-selective surface (FSS). The aim is to preserve or improve the absorption properties and at the same time to obtain radome transparency of the surface while keeping low RCS of the structure.

A common problem in electromagnetic design is to add frequency-dependent properties (here absorption, transparency) to a complicated, for example, a curved structure.
An example of such a complication is to, for example, investigate a large bandwidth frequency response of a layered structure by varying/optimizing layer thickness or periodic structures design. For planar surfaces, a unit-cell approach allows such an investigation with comparable short calculation time to evaluate many different designs towards approaching an optimum. A similar investigation for this case with its broken alignment between the unit cell that is now curved and the planar incoming wave makes a similar investigation considerably slower due to its larger electromagnetic size. It is known that applying a planar Jaumann absorber to a curved surface results in a certain performance loss [2], which can partly be compensated for on certain generic shapes like cylinders [3]. The design process of Jaumann-similar structure, for both the planar and cylindrical geometries, suffers from multiple local minimums in cost functions aiming to reduce their overall monostatic RCS behavior. Similar to, for example, [4], we address this challenge by first separating the design structure into several subfunction parts each optimized towards a "good" solution. Secondly, these partial designs are used as a starting point to fine-tune a global structure. It is shown in [1] that there are large similarities in the resonant frequency and absorption level between the planar 


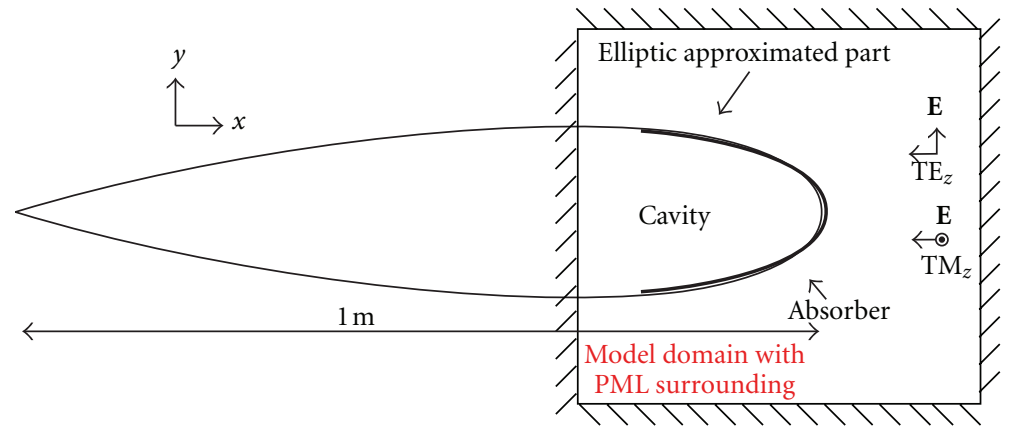

Figure 1: The cross-section of the aircraft wing profile. The simulated model is shown in the box surrounded by PML.

design and design on a surface with moderate curvature. This allows us here to use a planar model to investigate the possibility of adding a low-frequency transparency window to the structure by replacing a back-metal surface with a lowpass FSS, which is subsequently applied to a wing-front end to evaluate how this affect, its scattering properties.

The concept of a combined curved absorber with transparent window is not unique; in this paper, we combine a Jaumann absorber with a low-pass gangbuster-like FSS combined with a polarizer to add transparency in one polarization to a curved surface. Somewhat surprisingly we have not found any paper in the literature about this subject. The different subparts of the design are however well known, and a brief review of these is given here: the Salisbury screen [5], or the quarter wavelength absorber, which consists of a resistive sheet on top of a quarter wavelength thick dielectric spacer placed on a metal plane. It has a fairly narrow absorption bandwidth, which can be extended by adding additional resistive sheets and spacers; such a construction is commonly called a Jaumann absorber which is rather well known, see, for example, [4, 6, 7]. The Jaumann absorber has been extensively used to reduce RCS for the last half century. However, absorbers on nonplanar surfaces have considerably fewer references [1-3], each of these references was discussed above. Frequency-selective surfaces have been used to improve the absorption bandwidththickness efficiency of planar, see, for example, [8-11], where a periodic lossy FSS is used as a replacement of the resistive sheets. Another more extensively studied area is frequencyselective surfaces used as pass/stop band filters for a variety of polarizations, a review for planar surfaces, and a range of elements can be found in, for example, $[4,12]$.

The rest of the paper consists of Section 2 describing the design goals and constraints at our numerical model. A lowpass FSS is designed in Section 3. This designed FSS surface is integrated with the Jaumann absorber by replacing its metalback layer in Section 4. The section ends with a fine-tuning of the Jaumann parameters. A brief sensitivity analysis of the resulting planar structure is done in Section 5. In Section 6 is the designed structure applied to the wing front. The paper ends with conclusions and bibliography.

\section{Design Goals and Restrictions}

An infinitely extended cylinder with idealized wing crosssection is shown in Figure 1. The combined FSS absorber is applied to the front-end part. To emphasize the scattering properties of the applied absorbing/transparency structure, we further reduce the electromagnetic model to include only the $15 \%$ forward part of the wing. This front-end part is well approximated by the corresponding part of an ellipse with major and minor radius of $400 \mathrm{~mm}$ and $49.4 \mathrm{~mm}$, respectively. The resulting $15 \%$ structure is extended with a short block with a smooth transition from the elasticity curvature to horizontal ( $x$-parallel) and embedded in a PML layer to reduce back-end scattering. For a detailed discussion of the model and its implementation in Ansoft HFSS 11, see $[1,13]$. The applied structure is periodic in the $z$-direction. By utilizing a periodic (master/slave) boundary conditions, this allowed the final scattering structure to consist of a $6.8 \mathrm{~mm}$ thick ( $z$-direction) slice.

The frequency interval of interest here is $1-16 \mathrm{GHz}$. For $\mathrm{TM}_{z}$-polarized incoming waves (see Figure 1), the structure should reduce the monostatic RCS levels to below a metal wing RCS level over the entire frequency interval. Similar RCS reductions are required for incoming waves with frequencies between 2 and $16 \mathrm{GHz}$ in the $\mathrm{TE}_{z}$ polarization. In addition, the structure should be as transparent as possible to waves with $\mathrm{TE}_{z}$ polarization at $1 \mathrm{GHz}$. The long-term goal is to protect an antenna radiating $\mathrm{TE}_{z}$-polarized waves at $1 \mathrm{GHz}$. Such an antenna and its protecting structure will interact, and the radiation performance of the antenna, such as the level of polarization purity, increases its side lobe level, and transmission losses may be degraded, see, for example, $[14,15]$. All such considerations are beyond the scope of the present paper, since the antenna element is not yet determined. Starting from a concept-proved Jaumann absorber design [1], we replace its main wave-blocking feature, the metal-back plate, with an FSS surface. The design of such an FSS is the subject of the next section.

\section{A Gangbuster-Similar Low-Pass Frequency-Selective Surface}

To create a polarization-selective transparency window starting from a Jaumann absorber design, we note that its main blocking feature is the back-metal layer. Replacing the metal back with a suitable chosen FSS has the possibility to introduce transparency in one polarization at $1 \mathrm{GHz}$. However, the function of the Jaumann absorber strongly depends on the back metal for its function at its desired 


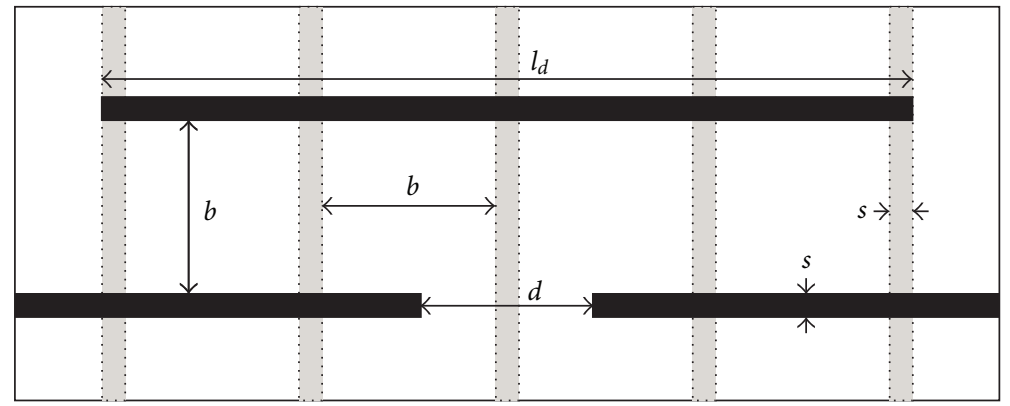

(a)

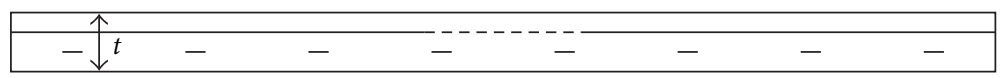

(b)

FIgURE 2: The design of the combined triangular lattice gangbuster together with the polarizer. The chosen values of the dimensions are given in the caption of Figure 3. The darker regions are metal, and the white region is dielectric. Each of the three dielectric spacers visible in (b) is $t / 3$ thick. (a) Top view of the $\left(d+l_{d}\right) \times(2 b+2 s)$ unit cell. (b) The cross-section of the FSS unit cell.

resonance frequency. To preserve as much of the backmetal properties as possible, and to introduce the desired separate scattering behavior of incoming $\mathrm{TM}_{z} / \mathrm{TE}_{z}$ waves, suggests that the choice of planar FSS structures should be in alignment with the polarizations for normal-incident waves. The frequency filter properties are strongly dependent on the patch type, its geometry, and size of the corresponding unit cell. A further limitation on the unit-cell requirement in our case is that the resulting design should be compatible with a one-direction periodicity requirement along the axis of the cylinder. A starting design for such a low-pass FSS compatible with the polarization requirements is an array of metal-patch dipoles from a family of low-pass frequencyselective surfaces called gangbusters $[4,16]$. To include the desired high-reflection behavior of the $\mathrm{TM}_{z}$ polarization, we combine such a gangbuster FSS with a periodic polarizer. A gangbuster FSS consists of straight dipoles arrayed in line with a small interdipole spacing, each line of dipoles is slightly displaced to the neighboring line, resulting in a nonrectangular unitcell. The gangbuster family has a lowpass behavior and for higher frequencies shows a wide-band high-reflection behavior; the family members mainly differ in size of the bandwidth of the low-pass region. To obtain a rectangular compatible unit cell, required for the simulation of a unit slice of the cylinder we modify a gangbuster of the "type 2" family (see [4]) to an equal-sides triangular lattice arrangement. We name the resulting triangular structure a gangbuster-like FSS, see Figure 2(a).

To combine the polarizer, consisting of long metal patches (vertical gray in Figure 2(a)), with the low-pass FSS metal patches (horizontal black) into one resulting structure, we stack them and embed both into a thin dielectric spacer (white), see Figure 2(b). This kind of embedding of gangbuster has been shown to work well in, for example, [4], with the additional benefit that the metal length becomes somewhat more frequency independent of the outside embedding dielectrics which are part of the Jaumann structure, see next section. Note that the respective

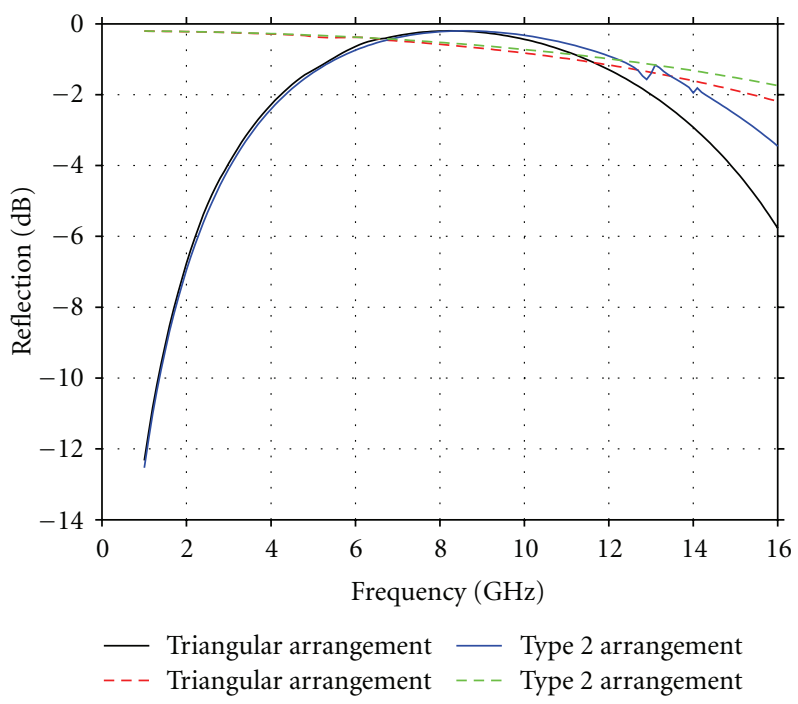

FIGURE 3: The reflection coefficient for a normally incident wave with parallel/orthogonal polarization upon the structure given in Figure 2. The dimensions correspond to $t=1.5 \mathrm{~mm}, d=3.0 \mathrm{~mm}, l_{d}$ $=8.4 \mathrm{~mm}, b=3.0 \mathrm{~mm}$ and $s=0.4 \mathrm{~mm}$, and $\varepsilon_{r}=2.2$. The reflection is compared with the performance of a gangbusters "type 2 " polarizer combination. Solid lines correspond to the parallel polarization and dashed lines correspond to orthogonal polarization, for example, where the $E$-field is parallel with dipole patches.

FSS layers are not in electrical contact with each other. The gangbuster family "type 2" [4] as well as our design has less than $12 \mathrm{~dB}$ reflection at $1 \mathrm{GHz}$. The resulting design parameters are given in the caption Figure 3.

The scattering performance of the lossless structure given in Figure 2 is shown in Figure 3; it is compared with the slightly better performing gangbuster "type 2" polarizer combination. Notice that the resulting structure reflects the orthogonal polarization, for example, where $E$-field is parallel to the polarizer and has low-pass characteristics for 


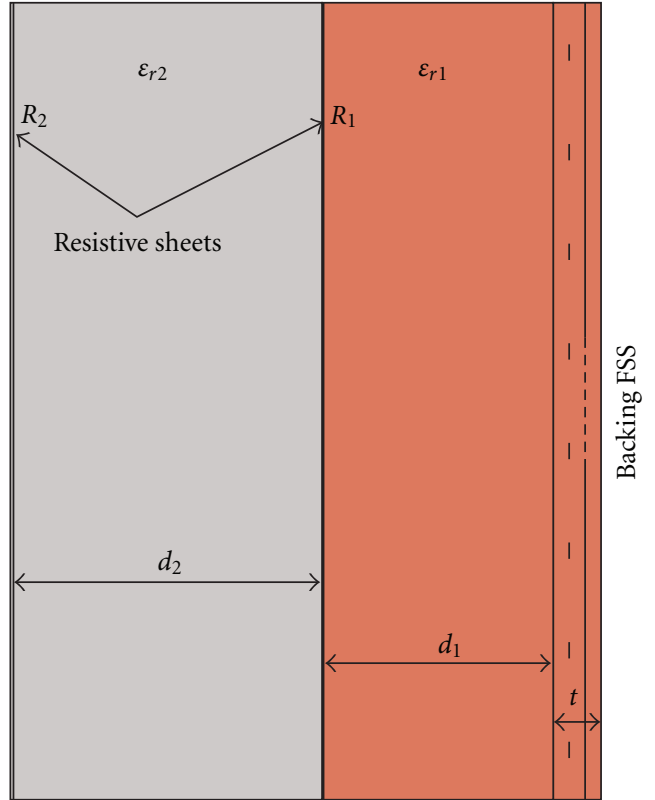

FIgURE 4: The cross-section of the FSS-backed Jaumann absorber.

the parallel polarization (dipoles parallel with $E$-field). As it is seen in Figure 3, we have obtained a high-reflection behavior for the orthogonal polarization over 1-16 GHz. For the parallel polarization, we have obtained a transparent window at and below $1 \mathrm{GHz}$ with less than $10 \mathrm{~dB}$ reflection (the structure is lossless). The frequency response then rapidly reaches towards a high-reflection behavior over frequency band between 3 and $15 \mathrm{GHz}$, with a normal reflection coefficient above $-4 \mathrm{~dB}$. The next step is to integrate the design given in Figure 2 with an absorber, which is the subject of the next section.

\section{An FSS-Backed Jaumann Absorber}

We have now obtained the first step in the design procedure, a frequency/polarization-sensitive structure with a lowfrequency "transparent" window and a high-frequency highreflection behavior, see Figure 3. The next step of the design process is to integrate this FSS structure as a replacement of the metal-back layer of a Jaumann absorber, see Figure 4

The goal here is to fine-tune the Jaumann parameters (spacer propertie, and the resistive layers) to optimize the survival of the $1 \mathrm{GHz} \mathrm{TE}_{z}$ polarization transparency while keeping the absorption properties of the original Jaumann absorber for both the $\mathrm{TM}_{z}$ polarization and for the 2$16 \mathrm{GHz}$ of the $\mathrm{TE}_{z}$ polarization. The lossy resistive layers of the absorber will couple the degree of the $\mathrm{TE}_{z}$ transmission at $1 \mathrm{GHz}$ with the absorbing properties for the same polarization in the $2-16 \mathrm{GHz}$ interval. We find naturally that a high transmission coefficient at low frequencies for the $\mathrm{TE}_{z}$ polarization degrades absorption at high frequencies and vice versa. Thus, the resulting structure will be a compromise between these two desired features. It is clear that incident waves scattering on this inhomogeneous structure containing the FSS surface will result in scattered wave in all directions. However, the small electric size of the unit cell in the computation frequency band, $16 \mathrm{GHz}$ and below, allows only evanescent higher-order Floquet modes. Thus, to tune the absorber properties, we make the approximation that the FSS layers can be replaced with an equivalent admittance response. This admittance is obtained from a numerical HFSS calculations of the reflection coefficient through the relation (see e.g., [17])

$$
Y_{\mathrm{FSS}}^{(\|, \perp)}=\frac{1}{Z_{\mathrm{FSS}}^{(\|, \perp)}}=Y_{0} \frac{1-\Gamma^{(\|, \perp)}}{1+\Gamma^{(\|, \perp)}} .
$$

Here, $Y_{0}$ is the intrinsic admittance of the free space and $\Gamma^{(\|, \perp)}$ is the reflection coefficient corresponding to parallel $\|$ or orthogonal $\perp$ polarization respectively, assuming that the cross polarizations is small. This approximation is used for optimization purposes to reach a compromise between transmission and absorption. In order to reach such a compromise we have studied a series of cost-functions as

$$
G(f)=\sum_{l=10}^{160} \max \left(\Gamma^{\|}\left(f_{0}+l \Delta f\right),-12\right)-w \mathrm{~T}^{\|}\left(f_{0}\right)
$$

Here $f_{0}=1 \mathrm{GHz}$ and $\Delta f=0.1 \mathrm{GHz}, \Gamma^{\|}$and $\mathrm{T}^{\|}$are the reflection and transmission coefficients, respectively, for the parallel polarization expressed in $\mathrm{dB}$ to emphasize the importance of the $1 \mathrm{GHz}$ transmission requirement. The relative weight between the higher frequencies and the transmission coefficient at $1 \mathrm{GHz}$ is controlled by the weight $w$. Notice that the cost function only contains the parallel polarization. This choice of $G$ enables fewer calculations resulting in a faster optimization procedure and the resulting design. The other polarization remains at least as good as the optimized one for frequencies above $3 \mathrm{GHz}$, see Figure 4 .

The cost function $G$ is minimized with respect to thicknesses and permittivities of each layer as well as the resistivity in each of the lossy layers (see Figure 4). The cost function $G$ suffers from a number of local minimums catching the used deterministic optimizer of $G$; to work around this problem we have used a range of starting conditions to improve the probability of finding a global optimum. We restricted the optimization domain of the parameters to:

$$
\begin{aligned}
2.2 \leq \varepsilon_{r 1} \leq 6, & 1.4 \leq \varepsilon_{r 2} \leq 6, \\
0.6 \leq R_{1} Y_{0} \leq 5, & 0.6 \leq R_{2} Y_{0} \leq 5, \\
2 \leq d_{1} \leq 7, & 2 \leq d_{2} \leq 7 .
\end{aligned}
$$

By varying the coefficient $w$ from 50 to 200, we get an almost linear variation of the $-10 \mathrm{~dB}$ absorption bandwidth from $10.1 \mathrm{GHz}$ to $8.8 \mathrm{GHz}$, and the corresponding transmitted power at $1 \mathrm{GHz}$ varies from $-4.94 \mathrm{~dB}$ to $-3.8 \mathrm{~dB}$, respectively. Thus, a more wide-band absorber can be achieved at the cost of a lower transmission at $1 \mathrm{GHz}$. A fast method to obtain the transmission/reflection coefficients for normal incident waves on a stratified structure is to use wave-splitting and propagation matrices for each layer, 
similarly to $[18,19]$. The resistive layers were considered to be sufficiently thin, so that they may accurately be accounted for as a generalized boundary condition [19].

The final design, using the homogenized FSS model, was obtained by using the weight $w=120$. The resulting parameters are $d_{1}=3.0 \mathrm{~mm}, d_{2}=5.7 \mathrm{~mm}, \varepsilon_{r 1}=2.2, \varepsilon_{r 2}=1.4, R_{1}=$ $414 \Omega / \square$, and $R_{2}=1260 \Omega / \square$. The corresponding reflection coefficients for the parallel and orthogonal polarizations are shown in Figure 5. The reflection coefficient of an optimized Jaumann absorber with the same total thickness (including the FSS thickness) as the optimized FSS jaumann is also shown in this figure. The Jaumann absorber is optimized with the same cost function as in (2) where the transmission term is eliminated. The resulting parameters for the Jaumann design are $d_{1}=4.4 \mathrm{~mm}, d_{2}=5.8 \mathrm{~mm}, \varepsilon_{r 1}=2.2, \varepsilon_{r 2}=$ 1.4, $R_{1}=228 \Omega / \square$, and $R_{2}=459 \Omega / \square$. The approximated design is compared with a full-wave simulation of the resulting structure which is presented by dotted lines for both polarizations. From the full-wave simulation, it is clear that the normal-incident reflection coefficient well agrees with the predicted homogenized result. We also find that the FSS Jaumann design provides desired level absorption over a large part of the frequency band. However, as it is expected, the absorption bandwidth is less than one obtained by an optimized metal-back Jaumann absorber with the same total thickness. It is due to the transparent window at low frequencies and the FSS thickness which is not used for the absorption means but for the transparency. More precisely, the $-10 \mathrm{~dB}$ absorption bandwidth for the orthogonal and parallel polarization is, respectively, reduced $8 \%$ and $17 \%$ for FSS-backed Jaumann as compared with the above-described optimized Jaumann. However, we can conclude that the metal-back replacement with a combined polarizer and a polarization-sensitive low-pass FSS results in a fair absorber. We are now in the position to investigate the transparency properties.

For the well-known power conservation relation for a homogeneous planar structure, see, for example, [20]

$$
P_{\text {loss }}=1-|\Gamma|^{2}-|T|^{2} \text {, }
$$

where $\Gamma$ and $T$ are the reflection and transmission coefficient of the homogenized FSS-backed Jaumann absorber, respectively, and $P_{\text {loss }}$ is the power loss of the structure, valid for normally incident plane waves of a lossy stratified nonmagnetic structure in free space.

We can now evaluate the level of transparency obtained by the resulting structure. The resistive losses of the structure at $1 \mathrm{GHz}$ are $43 \%$ which for this design could be lowered marginally on the expense of the absorption bandwidth. Thus, the FSS-backed Jaumann results in a partial transparency. However, rather large part of the $1 \mathrm{GHz}$ incoming field ends up as heat of the structure. What does this partial transparency imply for the RCS behavior of the curved surface?

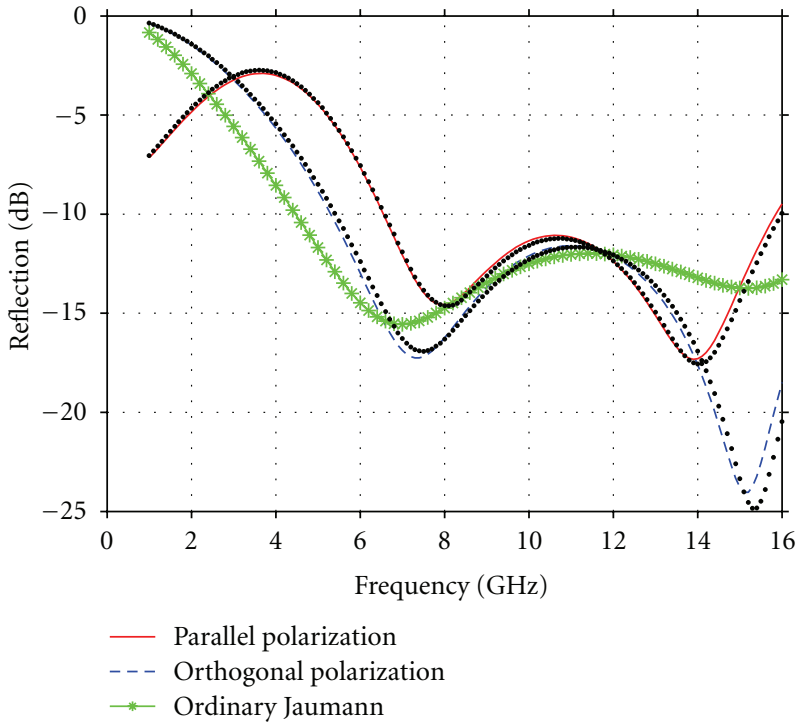

FIGURE 5: The reflection from the FSS-backed Jaumann absorber for the parallel (solid line) and orthogonal (dashed line) polarizations and the optimized Jaumann absorber with the same thickness (stars line) determined by the wave-splitting model. The full-wave analysis of each polarization is also shown by dotted-line curves.

TABLe 1: Sensitivity analysis of the structure shown in Figure 4. Here, $\left|\delta d_{i} / d_{i}\right| \leq 10 \%,\left|\delta R_{i} / R_{i}\right| \leq 10 \%, i=1,2$.

\begin{tabular}{lcccc}
\hline & $d_{1} \pm \delta d_{1}$ & $d_{2} \pm \delta d_{2}$ & $R_{1} \pm \delta R_{1}$ & $R_{2} \pm \delta R_{2}$ \\
\hline$|\delta \mathrm{BW} / \mathrm{BW}|$ & $<3.5 \%$ & $<0.4 \%$ & $<0.5 \%$ & $<2.2 \%$ \\
$\left|\delta T^{\|} / T\right|$ & $<0.2 \%$ & $<0.1 \%$ & $<6 \%$ & $<1.7 \%$ \\
\hline
\end{tabular}

\section{Sensitivity Analysis of the FSS-Backed Jaumann Structure}

In this section, we verify that the resulting design is robust towards small changes in the Jaumann parameters by a oneparameter variation of the design. The two quality evaluation parameters are the absorption bandwidth and the $1 \mathrm{GHz}$ transparency. Note that the relative thickness/dielectric properties cowork to make an electrical width of the dielectric spacers, thus to account for a combination of dielectricfrequency dependence and possible thickness variations it suffices to consider a range of thicknesses variations. It has been shown in [21] that the basic Jaumann absorbers are moderately insensitive to small changes in designed parameters. The similar response of the admittance representation of the FSS with the full-wave simulation allows us to use the simplified model to evaluate the robustness. Our perturbation study shows that our design is also moderately insensitive to small parameters perturbation. The results of the parameter variations are collected in Table 1.

Note that the largest sensitivity is in the levels of resistivity in $R_{1}$ changing the $1 \mathrm{GHz}$ transmission coefficient with $6 \%$. This indicates that it is necessary to include a lowpass behavior in at least one of the resistive layers. Work in this direction is in progress. 


\section{FSS-Backed Jaumann Absorber for an Aircraft Wing-Front Profile}

The question considered in this section is the following: does the FSS-backed Jaumann structure have an RCS lower than a plain metal wing-front when it is applied to the wing? The structure is applied on an elliptical layer in the wing front end by specifying the coordinates of each metal dipole patch of the FSS on an elliptic layer. Each layer in the FSS-backed Jaumann is mapped into a corresponding elliptical layer. The outer surface of the wing is replaced with the top-resistive layer $R_{2}$ of the FSS-backed Jaumann design. The dielectric space layer is created by inserting another elliptic surface which is shifted a distance $d$ in the $x$-direction. Each sequential layer of the design is similarly translated. The distance $d$ for each layer is identical with the corresponding thickness of the planar design $\left(d_{1}, d_{2}\right)$. This results in that each layer is very similar to the planar Jaumann along the $x$-axis, but the surface normal thickness away from the $x$-axis shrinks, resulting in a shift of frequency for wave of nonnormal incident direction. In [1], this was shown to be beneficial for the RCS reduction behavior for nonnormal incidence. The layer thicknesses are calculated in the optimization process for the planar structure and are equal to $3.0 \mathrm{~mm}$ and $5.7 \mathrm{~mm}$, respectively, for inner and outer layers. Similarly, the FSS structure is located inside a similar elliptical layer with maximal thickness of $1.5 \mathrm{~mm}$ at the front end. The wires are along $z$-axis, and the dipoles are bent along the elliptical curve of the wing. Thus, the parallel and orthogonal polarizations in the planar structure corresponds to the $\mathrm{TE}_{z}$ and $\mathrm{TM}_{z}$ polarizations in the curved structure with respect to the coordinate system reference shown in Figure 1 and the FSS in Figure 2.

Numerical simulations using HFSS were used to determine the mono-static RCS of a 6.8 slice "unit-cell" of an infinitely extended cylinder as described in Section 2. The wing is illuminated by an incident plane wave $\mathbf{E}^{i}$ with

$$
\mathbf{E}^{i}(x, y)=\mathbf{E}_{0} \exp \left[j k_{0}\left(x \cos \varphi_{i}+y \sin \varphi_{i}\right)\right],
$$

where $\varphi_{i}$ is the incident direction here $\varphi_{i}=0$, and $\mathbf{E}_{0}$ is either

$$
\mathbf{E}_{0}=E_{0} \hat{\mathbf{a}}_{z} \quad \text { or } \quad \mathbf{E}_{0}=E_{0}\left(-\hat{\mathbf{a}}_{x} \sin \varphi_{i}+\hat{\mathbf{a}}_{y} \cos \varphi_{i}\right),
$$

for the $\mathrm{TM}_{z} / \mathrm{TE}_{z}$ polarization, respectively. All fields are assumed to have the time dependence $e^{j \omega t}$.

There are now three interesting cases to compare for this wing-front end. The reference case is a plain metal-front end, which the designs are supposed to improve upon, while adding $1 \mathrm{GHz}$ transparency. This is the solid line in Figure 6. The second reference case is the optimized Jaumann absorber described in Section 4 (dashed-dotted curve in Figure 6). Both these reference cases are compared with FSS-backed Jaumann absorber. The dashed curve in the figure shows the performance of the FSS-backed Jaumann absorber. Notice that FSS-backed Jaumann absorber has an overall better performance in both polarizations than the metal-front end. The FSS-backed Jaumann provides a $-10 \mathrm{~dB}$ reduction of $2 \mathrm{D}$ RCS over $61 \%$ of the frequency band. It is observed that for

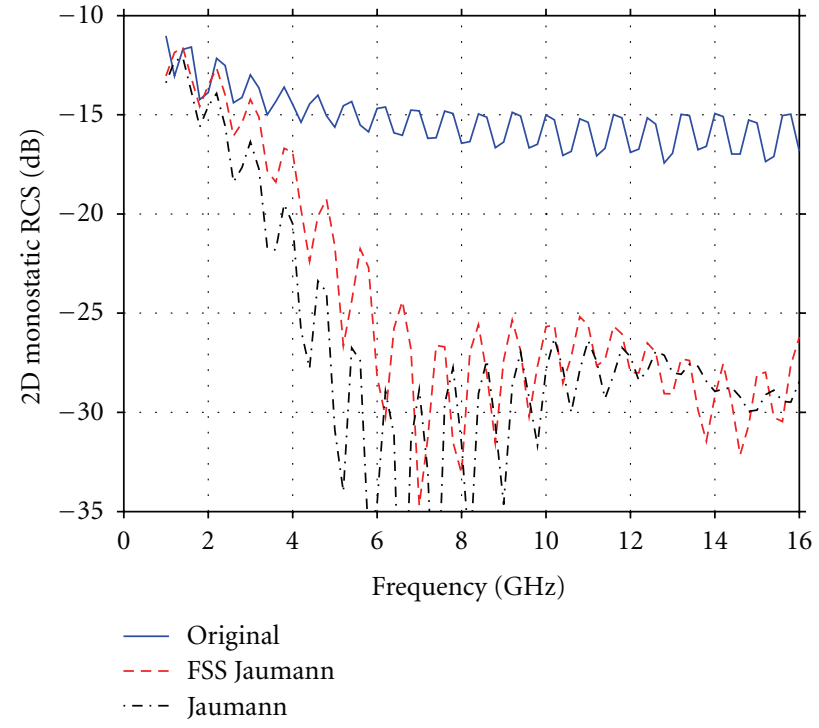

(a)

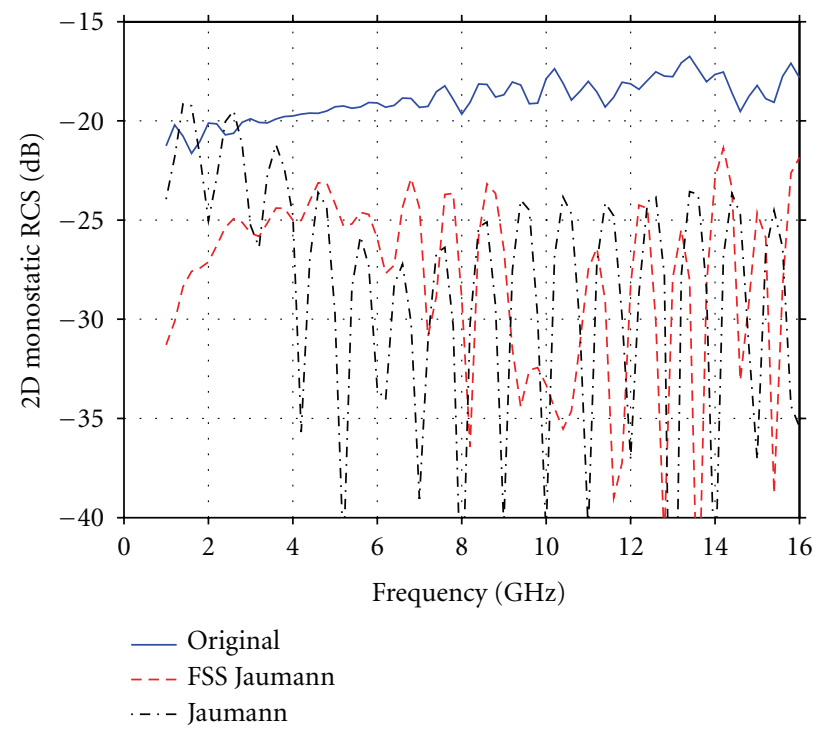

(b)

Figure 6: Monostatic RCS for normal-incident waves of the metalfront end of the wing (solid curve), Jaumann absorber front end of the wing (dashed-dotted curve), and FSS-backed Jaumann absorber front end of the wing (dashed curve). (a) shows the $\mathrm{TM}_{z}$ polarization, and (b) shows the $\mathrm{TE}_{z}$ polarization.

this polarization the RCS reduction performance of the FSSbacked Jaumann is slightly reduced as compared with the optimized Jaumann absorber.

The $9 \mathrm{~dB}$ improvement in RCS reduction at $1 \mathrm{GHz}$ for the $\mathrm{TE}_{z}$ polarization (Figure 6(b)) for the FSS-backed Jaumann absorber around $1 \mathrm{GHz}$ is clearly due to the transmission of the FSS surface. The cavity behind the FSS-backed Jaumann absorber has been modeled with a perfectly absorbing back wall, and hence all transmitted waves reaching the back wall are absorbed. Such absorption is used as a rough approximation on antenna absorption. The remaining part 
of the frequency band for the $\mathrm{TE}_{z}$ polarization is performing comparably or slightly degraded as compared with the optimized Jaumann absorber. We get a $5 \mathrm{~dB}$ reduction over $64 \%$ of frequency band. The absorption performance degradation of the FSS-backed Jaumann with respect to the optimized Jaumann is the cost of low-frequency transparency. It should be noted that both structures have the same total thickness. However, for the FSS-backed Jaumann, part of the thickness is used for the FSS while for the Jaumann all the thickness is used for absorption purpose. Since greater thickness allows higher absorption performance [22], the degradation of the absorption properties of the FSS-backed Jaumann with respect to the optimized Jaumann is expected. Furthermore, for $\mathrm{TE}_{z}$ polarization, part of this degradation is due to poor reflective properties of the FSS at low frequencies.

\section{Conclusion}

The investigation of planar absorption and transparency of the designed structure together with its RCS reduction behavior of a wing-front end shows that absorption persists in the presence of a backing FSS replacing the metal layer; a partial transparency is reached but with a high power loss, $43 \%$ at $1 \mathrm{GHz}$. The RCS reduction behavior of the structure degrades slightly as compared with an optimized Jaumann absorber with the same total thickness, applied to the curved surface. This degradation is due to the partial transparency of the structure at low frequencies. The inner resistive layer is through a perturbation analysis shown to have a large impact on the $1 \mathrm{GHz}$ transparency in the $\mathrm{TE}_{z}$ polarization. To reduce the transmission losses, a low-pass behavior of the resistive layers is required, and a continued investigation in this direction is in progress.

\section{Acknowledgments}

This work has been supported by The Swedish Governmental Agency for Innovation Systems (VINNOVA) within the NFFP4 SIGANT project. The authors also would like to thank SAAB Microwave Systems for their cooperation in this project.

\section{References}

[1] A. Motevasselian and B. L. G. Jonsson, "Radar cross section reduction of aircraft wing front end," in Proceedings of the IEEE International Conference on Electromagnetics in Advanced Applications (ICEAA '09), pp. 237-240, Turin, Italy, September 2009.

[2] E. F. Knott and K. B. Langseth, "Performance degradation of jaumann absorbers due to curvature," IEEE Transactions on Antennas and Propagation, vol. AP-28, no. 1, pp. 137-139, 1980.

[3] B. Chambers and A. Tennant, "Optimum design of cylindrical Jaumann absorbers," in Proceedings of the 9th International Conference on Antennas and propagations, pp. 450-454, 1995.

[4] B. A. Munk, Frequency Selective Surfaces, Theory and Design, Wiley, New York, NY, USA, 2000.

[5] W. W. Salisbury, "Absorbent body for electromagnetic waves," United States Patent 2,599,944, 1954.
[6] W. H. Emerson, "Electromagnetic wave absorbers and anechoic chambers through the years," IEEE Transactions on Antennas and Propagation, vol. AP-21, no. 4, pp. 484-490, 1973.

[7] L. J. du Toit, "Design of Jaumann absorbers," IEEE Antennas and Propagation Magazine, vol. 36, no. 6, pp. 17-25, 1994.

[8] A. K. Zadeh and A. Karlsson, "Capacitive circuit method for fast and efficient design of wideband radar absorbers," IEEE Transactions on Antennas and Propagation, vol. 57, no. 8, pp. 2307-2314, 2009.

[9] F. Sakran, Y. Neve-Oz, M. G. A. Ron, M. Golosovsky, D. Davidov, and A. Frenkel, "Absorbing frequency-selective-surface for the mm-wave range," IEEE Transactions on Antennas and Propagation, vol. 56, no. 8, pp. 2649-2655, 2008.

[10] O. Luukkonen, F. Costa, C. R. Simovski, A. Monorchio, and S. A. Tretyakov, "A thin electromagnetic absorber for wide incidence angles and both polarizations," IEEE Transactions on Antennas and Propagation, vol. 57, no. 10, pp. 3119-3125, 2009.

[11] F. Costa, A. Monorchio, and G. Manara, "Analysis and design of ultra thin electromagnetic absorbers comprising resistively loaded high impedance surfaces," IEEE Transactions on Antennas and Propagation, vol. 58, no. 5, pp. 1551-1558, 2010.

[12] R. Mittra, C. H. Chan, and T. Cwik, "Techniques for analyzing frequency selective surfaces-a review," Proceedings of the IEEE, vol. 76, no. 12, pp. 1593-1615, 1988.

[13] A. Motevsselian, On the scattering reduction of an aircraft wing profile enclosing an antenna, Licentiate thesis, Royal Institute of Technology (KTH), Stockholm, Sweden, 2010.

[14] E. Martini, F. Caminita, M. Nannetti, and S. Maci, "Fast analysis of FSS radome for antenna RCS reduction," in Proceedings of the IEEE Antennas and Propagation Society International Symposium, pp. 1800-1804, July 2006.

[15] H. Chen, X. Hou, and L. Deng, "Design of frequency-selective surfaces radome for a planar slotted waveguide antenna," IEEE Antennas and Wireless Propagation Letters, vol. 8, pp. 12311233, 1980.

[16] S. W. Schneider and B. A. Munk, "The scattering properties of super dense arrays of dipoles," IEEE Transactions on Antennas and Propagation, vol. 42, no. 4, pp. 463-472, 1994.

[17] D. M. Pozar, Microwave Engineering, Addison Wesley, 2000.

[18] J. A. Kong, Electromagnetic Theory, Wiley, New York, NY, USA, 1985.

[19] E. F. Knott, J. F. Shaeffer, and M. T. Tuley, Radar Cross Section, Artech House, Boston, Mass, USA, 1993.

[20] C. Sabah and S. Uçkun, "Electromagnetic wave propagation through frequency-dispersive and lossy double-negative slab," Opto-Electronics Review, vol. 15, no. 3, pp. 133-143, 2007.

[21] A. Charles, M. S. Towers, and A. McCowen, "Sensitivity analysis of Jaumann absorbers," IEE Proceedings: Microwaves, Antennas and Propagation, vol. 146, no. 4, pp. 257-262, 1999.

[22] K. N. Rozanov, "Ultimate thickness to bandwidth ratio of radar absorbers," IEEE Transactions on Antennas and Propagation, vol. 48, no. 8, pp. 1230-1234, 2000. 

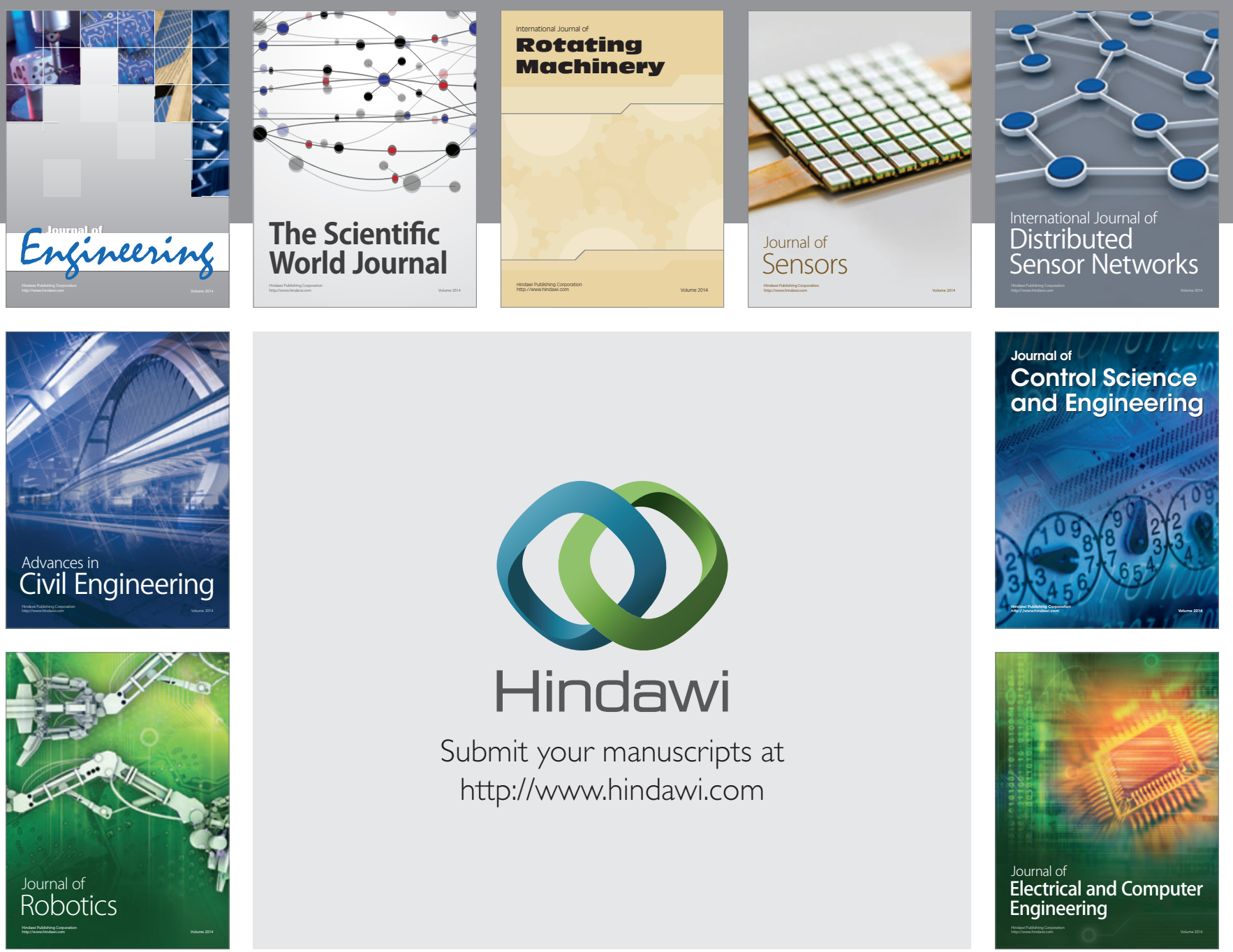

Submit your manuscripts at

http://www.hindawi.com
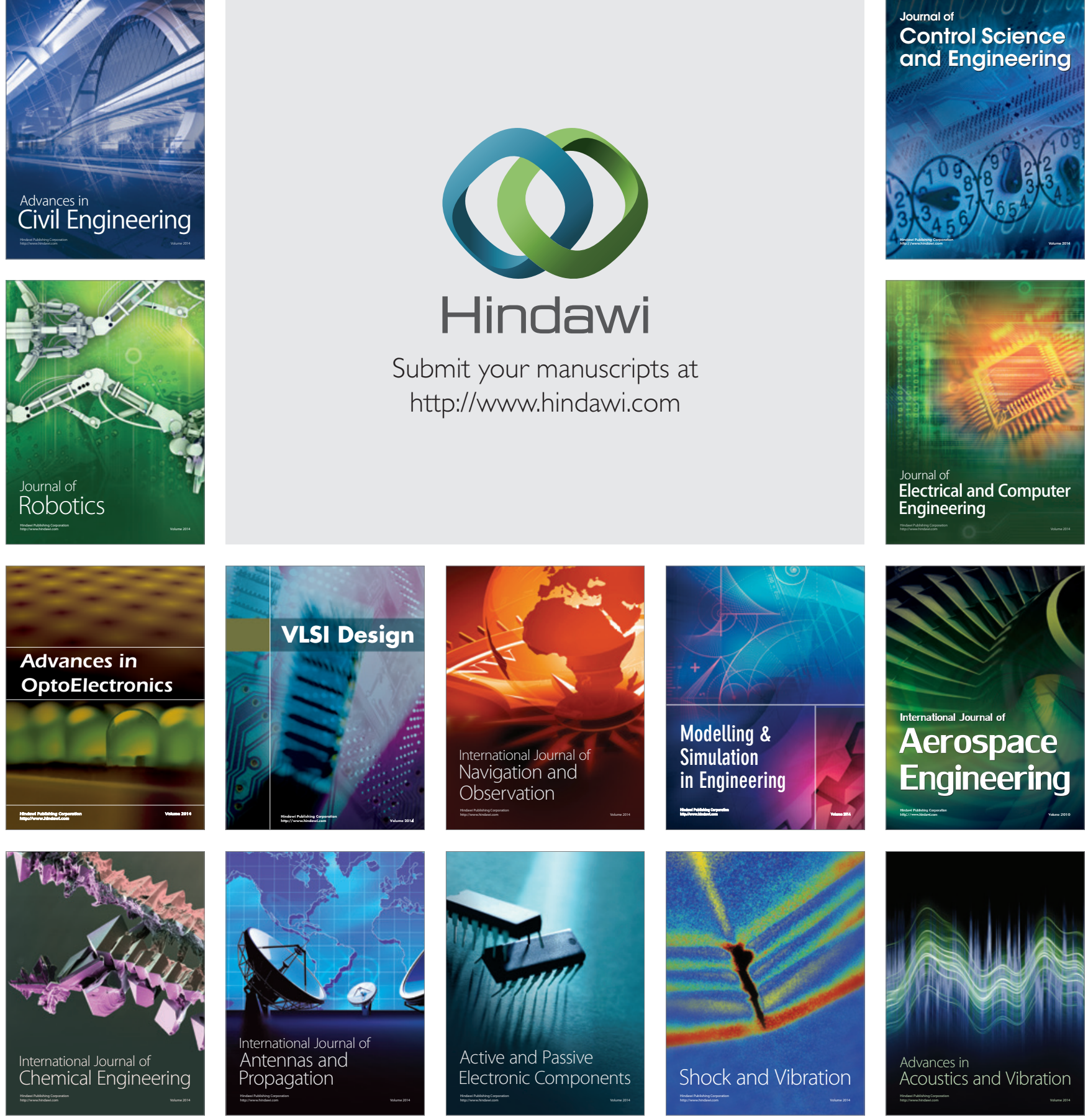